\title{
Linear Fractional Stable Motion with the rlfsm R Package
}

\author{
by Stepan Mazur and Dmitry Otryakhin
}

\begin{abstract}
Linear fractional stable motion is a type of a stochastic integral driven by symmetric alpha-stable Lévy motion. The integral could be considered as a non-Gaussian analogue of the fractional Brownian motion. The present paper discusses $\mathrm{R}$ package rlfsm created for numerical procedures with the linear fractional stable motion. It is a set of tools for simulation of these processes as well as performing statistical inference and simulation studies on them. We introduce: tools that we developed to work with that type of motions as well as methods and ideas underlying them. Also we perform numerical experiments to show finite-sample behavior of certain estimators of the integral, and give an idea of how to envelope workflow related to the linear fractional stable motion in S4 classes and methods. Supplementary materials, including codes for numerical experiments, are available online. rlfsm could be found on CRAN and gitlab.
\end{abstract}

\section{Introduction}

The linear fractional stable motion (shortly, lfsm) $\left(X_{t}\right)_{t \in \mathbb{R}}$ on a filtered space $\left(\Omega, \mathcal{F},\left(\mathcal{F}_{t}\right)_{t \in \mathbb{R}}, \mathbb{P}\right)$ is defined via

$$
X_{t}=\int_{\mathbb{R}}\left\{(t-s)_{+}^{H-1 / \alpha}-(-s)_{+}^{H-1 / \alpha}\right\} d L_{s}, \quad x_{+}:=\max \{x, 0\}
$$

where $L_{s}$ is a symmetric $\alpha$-stable Lévy motion, $\alpha \in(0,2)$, with the scaling parameter $\sigma>0$ and the self-similarity parameter $H \in(0,1)$. The lfsm is heavy-tailed process with infinite variance and long-range dependence. A good overview on the role which this process plays in natural sciences is done by Watkins et al. (2008). One could also find a review of stochastic properties of lfsm in Mazur et al. (2020).

We proceed with introduction to existing software, with interest towards study of numerical properties of statistical estimators for lfsm as the main motivation. So far, there is no standard approach for software development to operating the general class of stochastic processes driven by Lévy processes. Moreover, there was no systematic indexed and pier-reviewed software for simulating sample paths of lfsm and related estimators prior to rlfsm. There is a particularly simple and useful numerical algorithm for simulating lfsms developed by Stoev and Taqqu (2004). Other methods for simulation of the processes can be found in (Wu et al., 2004) and (Biermé and Scheffler, 2008). The paper (Stoev and Taqqu, 2004) contains a minimalistic implementation of lfsm generator as a MATLAB function. However, some useful packages, that could be used in numerical routines with Lévy-driven processes (e.g. to create lfsm generator and perform unit testing), exist and have been implemented in R. For instance, R package somebm (Huang, 2013) contains functions for generation of fractional Brownian motion (fBM). Currently archived by CRAN dvfBm (Coeurjolly, 2009) has routines for generation of $\mathrm{fBm}$ and estimator of the Hurst parameter of the latter. stabledist (Wuertz et al., 2016) and stable (Swihart et al., 2017) contain different functions for stable distributions and random variables. A generator of random variables of the kind has been also implemented in MATLAB (see the code in Chapter 1.7 in (Samorodnitsky and Taqqu, 1994)).

The paper is organized as follows. In Section 2.2 we present the simulation method for sample paths of lfsm and its implementation in our path function. Then, we present functions for finite sample studies of statistical estimators, and some other functions. Section 2.3 describes implementations of the high- and the low-frequency parameter estimators and discusses reasons behind their numerical behavior. Finally, in Section 2.4 we suggest an object oriented system that simplifies software programming of Lévy-driven integrals.

\section{Basic R functions}

\section{Types of data we use}

The latest version of the package (1.0.0) suggests that we work with two types of sample paths. In the low-frequency setting we only use points spaced 1 temporal index apart from each other, $X_{1}, X_{2}, \ldots, X_{n}$. In the case of high-frequency, we use points with discretization equal to the length of the path vector, $X_{1 / n}, X_{2 / n}, \ldots, X_{1}$. This division is dictated by two issues: 1$)$ the same division 
in the setting of limit theorems obtained by Mazur et al. (2020), and 2) the fact that there is no inference technique for an arbitrary mixture of the two frequencies. Consequently, temporal coordinates of low-frequency lfsm coincide with point index (compare coordinates and point_num in the example in Section 2.2.2) which varies from 0 to $N$. Analogously, in case of high-frequency scheme, temporal coordinates equal to point indexes divided by the total number of sampled points. When after sampling the index set is different from either $(1,2, \ldots, N)$ or $(1 / n, 2 / n, \ldots, 1)$, rescaling in time should be performed using the equality $\left(a^{H} X_{t}\right)_{t \geq 0} \stackrel{d}{=}\left(X_{a t}\right)_{t \geq 0}$ with $a>0$ provided that $H$ is known or obtained via preliminary estimation.

\section{Simulation method for the linear fractional stable motion}

In this section, we start with a discussion on the simulation method of the lfsm proposed by Stoev and Taqqu (2004) which is implemented in R by us. In particular, simulation of sample paths is done via Riemann-sum approximations of its symmetric $\alpha$-stable stochastic integral representation while Riemann-sums are computed efficiently by using the Fast Fourier Transform algorithm. In R, we introduce path function that creates sample paths of the lfsm. The idea underlying this sample path generator is that it should be always possible not only to obtain lfsm path, but also the underlying Lévy motion, generated during the procedure, and since the core function of lfsm is deterministic it should allow for lfsm path generation based on a given Lévy motion, and, in theory, otherwise (not always). For this reason generators of both processes were separated into independent parts (see Figure 1).

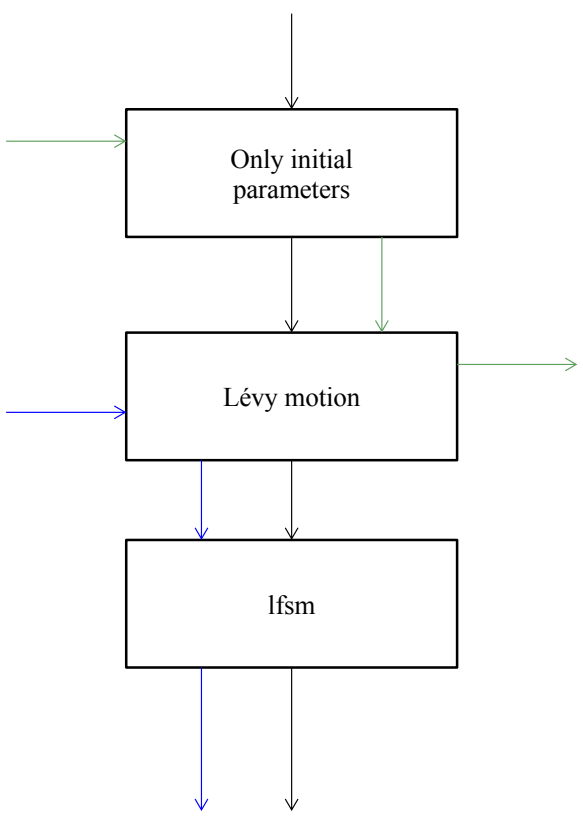

Figure 1: Scheme of generating Lévy motion and lfsm by path. Black arrows: when the algorithm initially is given the parameters, it generates Lévy motion, and then lfsm. Green arrows: when Lévy motion is needed without lfsm in order to save processing time, the algorithm bypasses computing of the later. Blue arrows: given a Lévy motion and some parameters, the generator computes the corresponding lfsm.

The function path can be used by

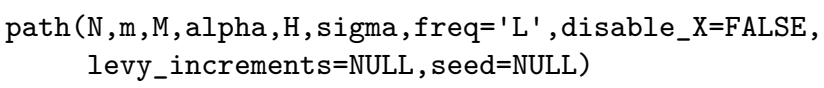

Parameters N,m, M regard to the index of the process, or time, if applicable. $m$ and $M$ are the only means to control precision of the integral computation. $\mathrm{N}$ is a number of points of the lfsm to generate. $\mathrm{m}$ is a discretization parameter that corresponds to the number of points where Lévy motion is sampled between two nearby indexes (e.g. $N$ and $N-1$ ). M is the truncation parameter, i.e. number of points after which the integrated function is set to zero; freq stands for the frequency of the motion which can take two values: ' $\mathrm{H}$ ' for high-frequency and ' $\mathrm{L}$ ' for the low-frequency setting. This is the switch between the two data types. disable_X is needed to disable computation of $\mathrm{X}$, 
the default value is 'FALSE', when it is 'TRUE', only a Lévy motion is returned, which in turn reduces the computation time. seed is a parameter that performs seeding of the lfsm generator. Technically, in the path the seed is set just before Lévy increments are generated. The path function returns a list containing the lfsm, the underlying Lévy motion, the point number of the motions from 0 to $N$ (point_num) and the corresponding coordinate which depends on the frequency, the parameters $(\sigma, \alpha, H)$ that were used to generate the lfsm, and the predefined frequency.

Generation of symmetric $\alpha$-stable ( $\mathrm{s} \alpha \mathrm{s})$ random variables is powered by function rstable from package stabledist with SO parametrization based on the Zolotarev's representation for an $\alpha$-stable distribution with some modifications. So is used in order to make sigma a scale parameter of the motion and to get exempt from computing the normalization constant $C_{H, \alpha}$ presented in Stoev and Taqqu (2004) and is given by

$$
C_{H, \alpha}:=\left(\int_{\mathbb{R}}\left|(1-s)_{+}^{H-1 / \alpha}-(-s)_{+}^{H-1 / \alpha}\right|^{\alpha} d s\right)^{1 / \alpha}
$$

\section{The discrete convolution based algorithm and particularities of indexing}

As it was mentioned in the beginning of Section 2.2.2, one of the features of path is the ability to operate on a pair lfsm - Lévy motion and to switch between them. We recall that direct computation of the sum approximating the integral in the definition of lfsm (1) would involve number of operations proportional to $N M m$, which makes the method slow. Instead, the original algorithm by Stoev and Taqqu (2004) suggests computing increments of lfsm with the help of

$$
W(n):=\sum_{j=1}^{m M} a_{H, m}(j) Z_{\alpha}(n-j),
$$

where $W(m k)$ is a discretized and truncated version of the increments of the lfsm, and in the limit has the same distribution as them

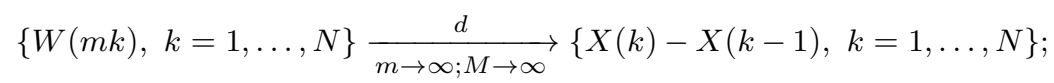

$Z_{\alpha}(k)$ are i.i.d. $\mathrm{s} \alpha \mathrm{s}$ random variables that have indexes $-m M, \ldots, m N-1$ and scaling parameter equal to 1 , and

$$
a_{H, m}(j):=C_{H, \alpha}^{-1}(m, M)\left((j / m)^{H-1 / \alpha}-(j / m-1)_{+}^{H-1 / \alpha}\right) m^{-1 / \alpha}, j \in \mathbb{N}
$$

\begin{tabular}{|c|c|c|c|c|c|c|c|c|}
\hline-2 & -1 & 0 & 1 & 2 & 3 & 4 & 5 & index \\
\hline 7 & 9 & 1 & 3 & 8 & 2 & 6 & 8 & z \\
\hline & & 4 & 9 & 2 & & & & a \\
\hline
\end{tabular}

with

$$
C_{H, \alpha}(m, M):=m^{-1}\left(\sum_{j=1}^{m M}\left|(j / m)^{H-1 / \alpha}-(j / m-1)_{+}^{H-1 / \alpha}\right|^{\alpha}\right)^{1 / \alpha}
$$

Figure 2: Example of direct computation of sum of the form (2) for 2 vectors. $a$ corresponds to the kernel and $Z$ - to the Lévy motion.

Let us consider an example which will recur and evolve throughout this section. Consider computing sum (2) where $m=1, M=3$, and $N=6$ (see Figure 2). The two rightmost cells for $W(n)$ are left empty because there is no sense in computing them without truncation of $a$.

A method based on the discrete convolution theorem is used to obtain $W(m k)$. The theorem relies on Discrete Fourier Transform (DFT), which needs to perform a number of operations proportional to $(m N+m M) \log (m N+m M)$ instead of $N M m$. In order to understand how this method works, we review several definitions and theorems.

Definition 2.2.1 For any sequence $x_{n}, n \in \mathbb{N}$, Discrete-Time Fourier Transform (DTFT) is defined as

$$
X=\operatorname{DTFT}\left\{x_{n}\right\}(\omega)=\sum_{n=-\infty}^{\infty} x_{n} \exp (-i n \omega) .
$$


The reverse transform, IDTFT, is defined as

$$
x_{n}=\operatorname{IDTFT}\{X\}=\frac{1}{2 \pi} \int_{0}^{2 \pi} X(\omega) e^{i \omega n} d \omega .
$$

Definition 2.2.2 Discrete convolution of two infinite sequences $\left\{A_{n}\right\}_{n \in \mathbb{N}}$ and $\left\{B_{n}\right\}_{n \in \mathbb{N}}$ is

$$
(A * B)[n]:=\sum_{m=-\infty}^{\infty} A[m] B[n-m] .
$$

There is a convolution theorem for discrete sequences which says that the discrete convolution of two sequences is equal to the Inverse Discrete Fourier Transform (IDFT) of the multiplication of the direct transforms of the sequences:

Theorem 2.2.3 For any discrete sequences $x_{n}$ and $y_{n}, n \in \mathbb{N}$, it holds that

$$
(x * y)[n]=\operatorname{IDTFT}\left[\operatorname{DTFT}\left\{x_{n}\right\}(\cdot) \times \operatorname{DTFT}\left\{y_{n}\right\}(\cdot)\right] .
$$

Definition 2.2.4 Let $x_{n}, n \in \mathbb{N}$ be a sequence. Then $\left\{x_{N}\right\}[n], n \in \mathbb{N}$ is called $N$-periodic summation of the sequence:

$$
\left\{x_{N}\right\}[n]:=\sum_{k \in \mathbb{N}} x[n+k N]
$$

It is straightforward that the periodic summation in the definition above has period $N$. In our case, the latter theorem is applicable even though we will be interested in a finite sequence of length $\tilde{N}$. The sequence is padded with zeros to form an infinite one, and a periodic summation of a the length $\tilde{N}$ is just a periodic extension of it.

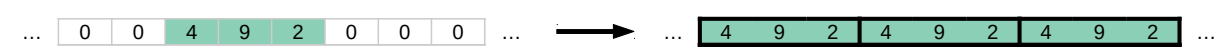

Figure 3: Example of periodic summation of a zero-padded finite sequence where the period equals to the sequence length $(N=\tilde{N})$.

DTFT is not directly useful for simulation purpose, that is why we need a special case of Theorem 2.2.3, Circular Convolution Theorem which reduces DTFT to DFT.

Definition 2.2.5 The DFT of a finite sequence $x_{n}$ of length $N$ is defined as

$$
X_{k}=\operatorname{DFT}_{k}\left(x_{n}\right):=\sum_{n=0}^{N-1} x_{n} \exp (-2 \pi i k n / N) .
$$

The IDFT is

$$
x_{n}:=\frac{1}{N} \sum_{k=0}^{N-1} X_{k} \exp (2 \pi i k n / N) .
$$

Theorem 2.2.6

$$
\left(x_{N} * y\right)[n]=\operatorname{IDFT}\left\{\operatorname{DFT}\left(x_{N}\right) \operatorname{DFT}\left(y_{N}\right)\right\}
$$

Returning to the task of computing the sum in (2), we consider two vectors: $a$ of length $m M$ and $Z$ of length $m(M+N)$. Here, we again index vectors starting with zero, not one. If we extend $Z$ periodically, pad $a$ with zeros to make an infinite sequence, and compute $\left(a * Z_{m(N+M)}\right)[n]$, values with indexes $[m M ; m(N+M)-1]$ would coincide with the result of a convolution of $a$ and $Z$. The first $m M$ values would be meaningless. This gives an idea how to use Circular Convolution Theorem for computation of (2): instead of $a * Z$ we compute one period of $\left(a * Z_{m(N+M)}\right)[n]$ through the left part of 2.2.6 and leave only meaningful values. Figure 4 illustrates the use of Circular Convolution Theorem with periodic extensions of $Z$ and padded $a$ to compute (2). In this case results with indexes -1 and -2 are meaningless and should be discarded.

Although the setup of the example as is on Figure 4 is fastest, it is impossible to use it directly, because in some situations truncation parameter $M$ is larger than $N$, the number of points of lfsm 


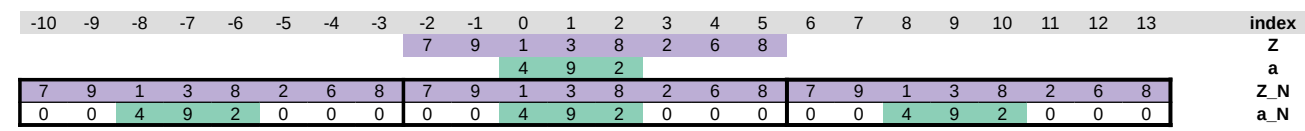

Figure 4: Example of transformation of vectors $a$ and $Z$ into sequences before computing their convolution.

sample path that is needed to be simulated. In this case path function performs an index shift using the following property:

$$
\begin{aligned}
\left(a * x_{c}\right)[n] & :=\sum_{k=-\infty}^{+\infty} a[k] \cdot x[n-k-c] \\
& =\sum_{k=-\infty}^{+\infty} a[k] \cdot x[\tilde{n}-k]=(a * x)[\tilde{n}-c]
\end{aligned}
$$

This property is illustrated by Figure 5 , wherein sequence $x[n]$ is shifted by 2 to the right, so $c=2$. Accordingly, the resulting convolution also gets shifted 2 notches to the right (compare Figures 5 and 2). In general, according to (3), when $x[n]$ is shifted to assign index zero to the first value, the resulting convolution sequence also starts from the first meaningful value. Thus, path always keeps the first $N m$ as the result of convolution operation and discards the rest.

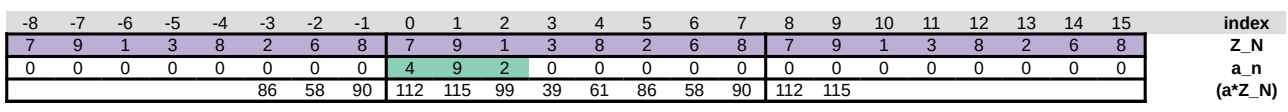

Figure 5: Example of index shift in path function.

\section{Examples}

In the next example, we show how one can use the above function to generate a sample path and to provide its visualization. Compare the procedure with the similar one from Section 2.4.1.

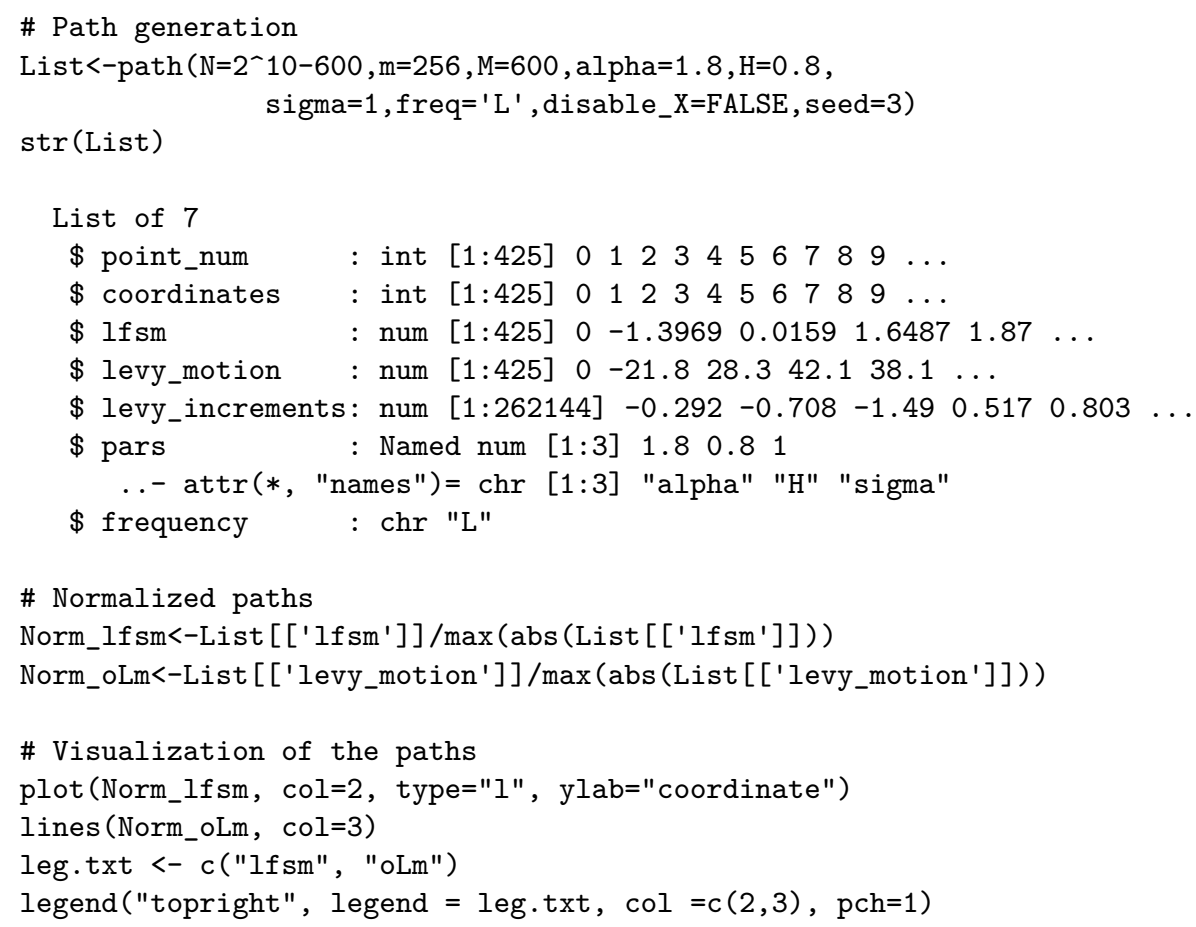

The result of the chart rendering is shown on Figure 6. The following example shows how to switch path function in order to alter between simulation of lfsm from scratch and computing based on an existing sample path of the Lévy motion. 


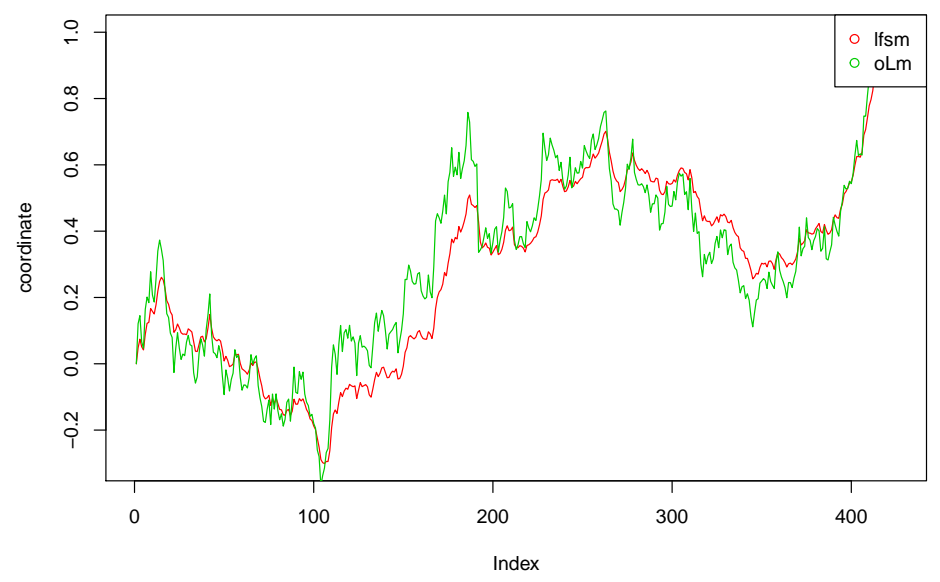

Figure 6: Plot of sample path and Lévy motion with seed=2

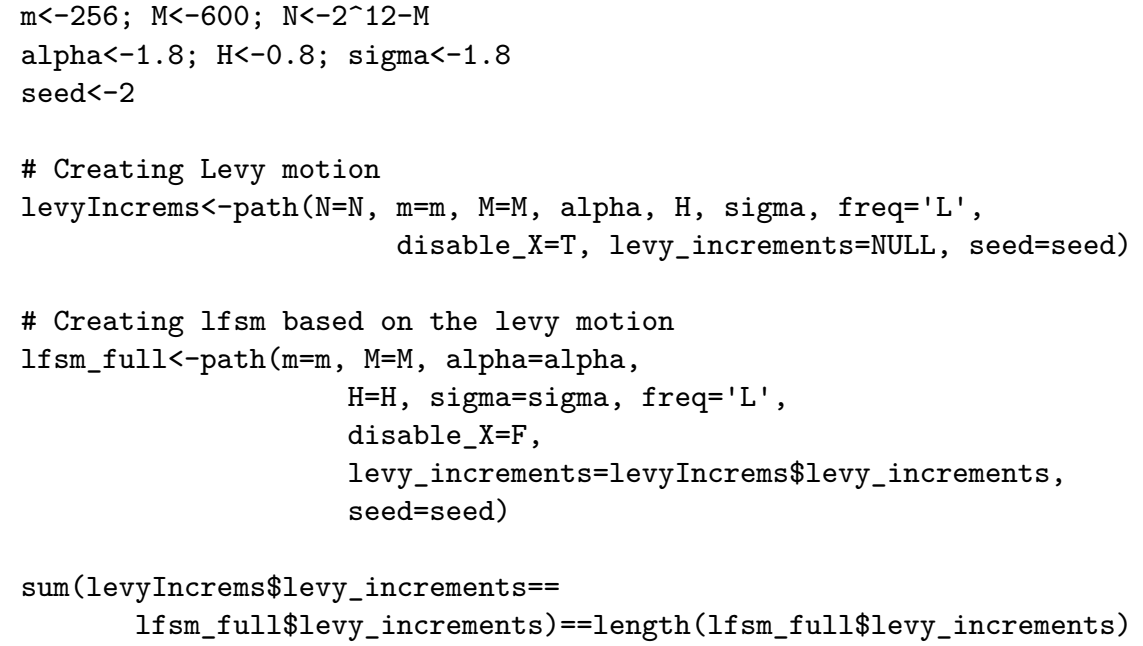

\section{[1] TRUE}

In the example the Lévy motion is generated without computing the lfsm, which was done by setting disable_X=TRUE, and saved to variable levyIncrems. After that, path was given the obtained Lévy increments and, basing on them, generated an lfsm path. As one can observe, the Lévy increments from the both objects produced by path are identical. The same holds when we obtain an lfsm path from the above procedure and one-step simulation of lfsm with seeding. These two facts are used in automated tests provided for rlfsm package.

\section{MCestimLFSM and numerical properties of statistical estimators}

In order to study numerical properties of the estimation procedures developed in Mazur et al. (2020), we created a technique, that could be used in solving this problem for any pair stochastic process and an estimator. The approach was implemented in MCestimLFSM function (Figure 9). The main motivation here is that for some estimators we have limit theorems, but we do not have theory which describes estimator behavior when the length of a path is relatively small, and thus, for instance, we cannot use closed-form expressions to obtain confidential intervals. In the following examples we show how to use functions MCestimLFSM, PLot_vb, and Plot_dens for studying empirical variance, bias and a density function of an estimator. In the first example, we study GenLowEstim estimator, and its bias and variance dependencies on the length of the sample paths. In particular, one would be able to determine starting from which path length the estimator loses significant bias influence. 


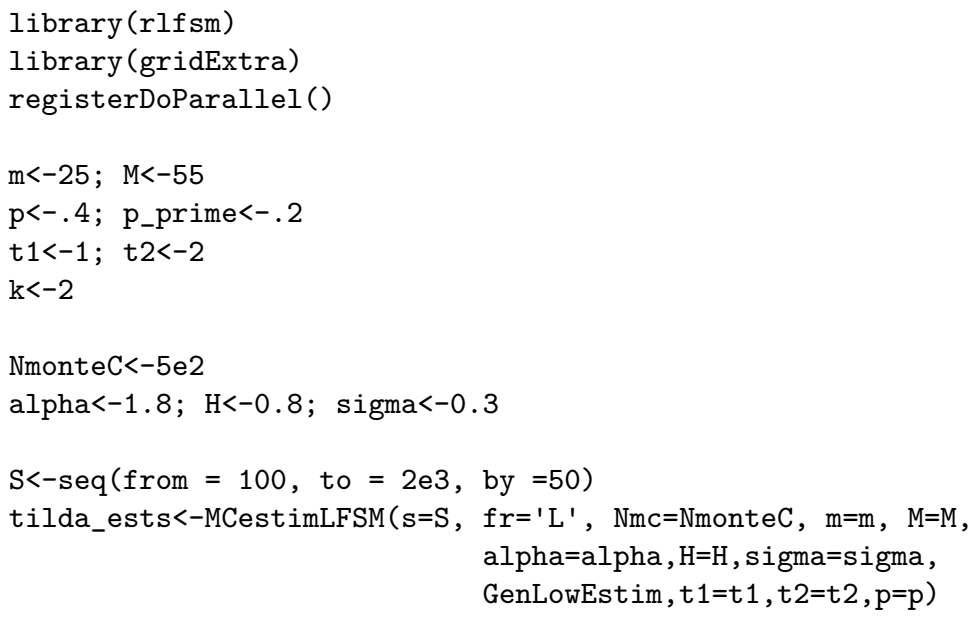

Plot_vb(tilda_ests) 


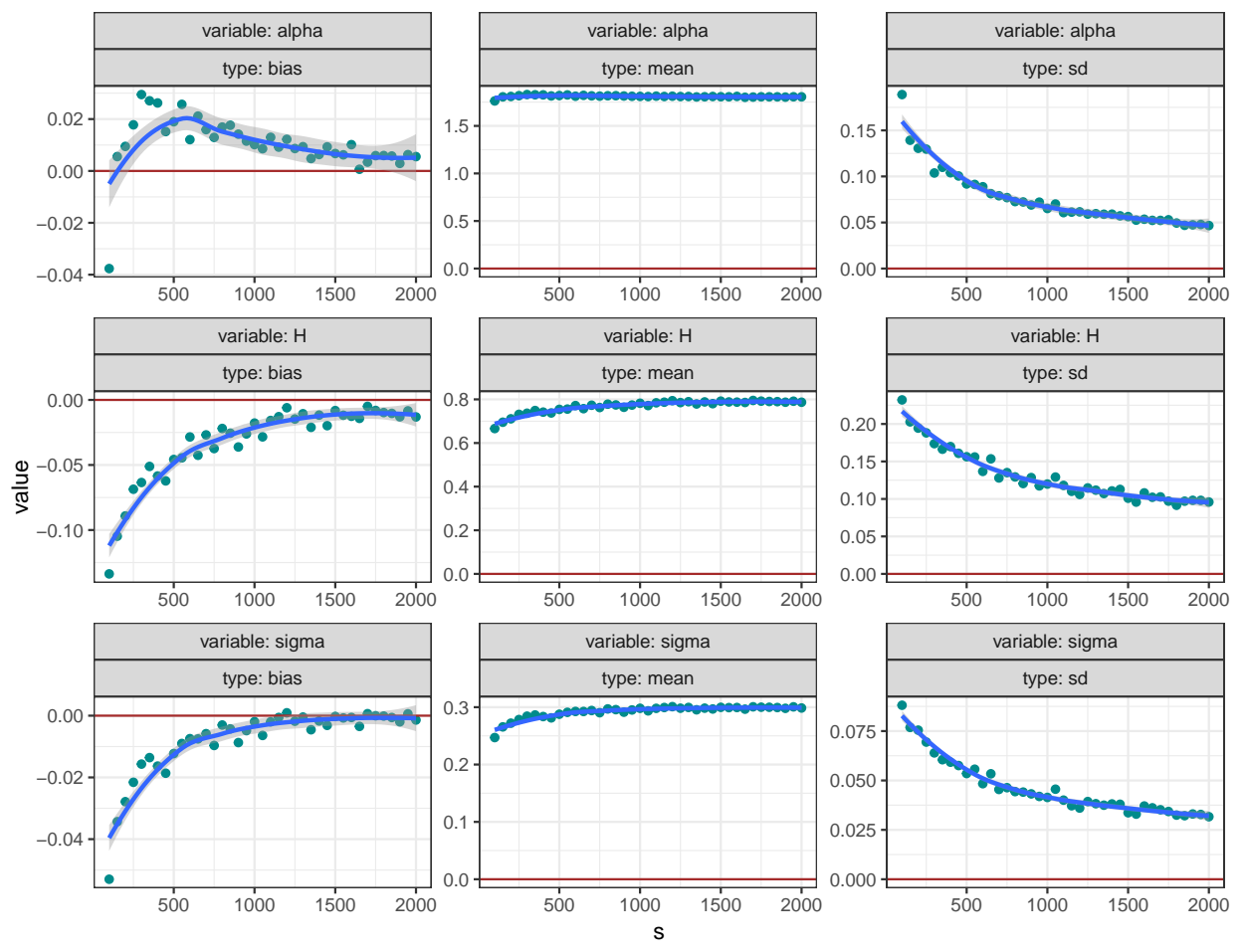

Figure 7: Variance and bias dependence on path length of tilde- estimators, described in Section 2.3.2.

Figure 7 shows that when $(\sigma, \alpha, H)=(0.3,1.8,0.8)$, estimator GenLowEstim could be considered unbiased starting approximately from 1000 points.

The second example compares empirical standardized densities of estimates, obtained by GenLowEstim with the limiting standard normal ones, Figure 8.

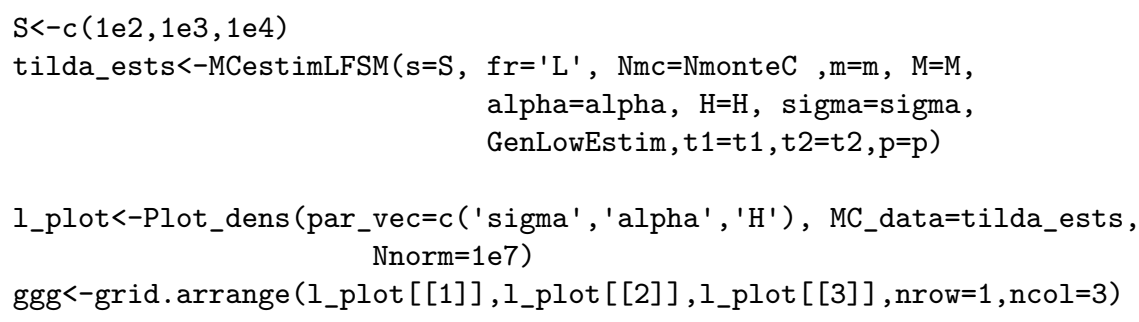

In short, in these examples for different path lengths s, NmonteC lfsm paths are simulated. To each path we apply tilde-statistic (see Section 2.3.2), therefore obtaining NmonteC estimates $\left(\tilde{\sigma}_{\text {low }}, \tilde{\alpha}_{\text {low }}, \tilde{H}_{\text {low }}\right)$ for every $s$, which in turn, are used to calculate biases, standard deviations, and density functions (also, for each $s$ separately).

\section{MCestimLFSM architecture and optimization}

It is important to notice that generation of lfsm is numerically heavy routine and also a large number of estimates is needed to compare their empirical distributions with the limiting ones. The latter task gave MCestimLFSM its name. Thus, in order to make computations feasible in terms of time and memory use, the architecture of MCestimLFSM must be well-optimized. Apparently, a multi-core setup is crucial for dealing with the task.

Having fixed a path length, the whole procedure behind MCestimLFSM could be split in two parts. First, we need to obtain samples for each estimator. Second, we obtain statistics of these samples (see Figure 9). Once finished, MCestimLFSM proceeds to the next length value until reaches the end of the vector of lengths.

In the first part, we generate $N_{\text {Monte Carlo }}$ lfsm paths of the length s [i] via path_fast function. To each of the paths we apply all the estimators to obtain $H, \alpha$, and $\sigma$ estimates. During this stage, we use a foreach-based parallel loop, where each node simulates a path, computes and returns the statistics removing the path from memory. path_fast is an unavailable for users version of 

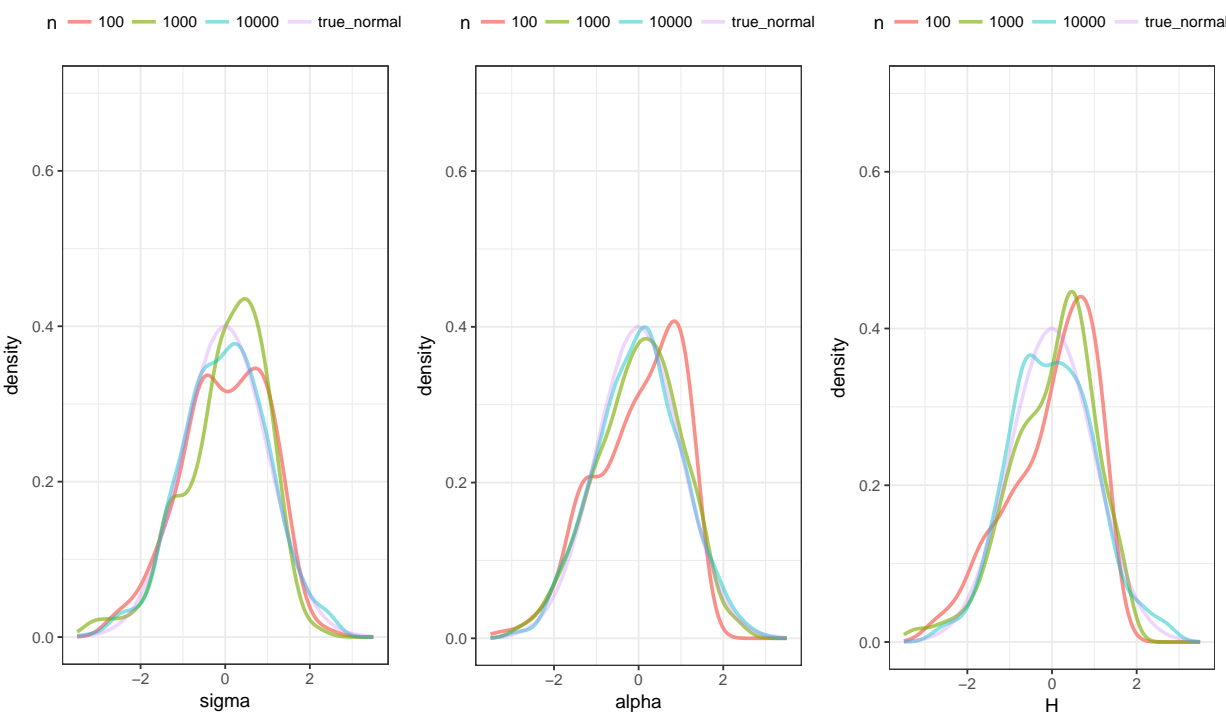

Figure 8: Empirical distributions of tilde- estimates, described in Section 2.3.2.

path with significantly reduced functionality for the sake of saving execution time. The further desired enlargement of the node task by adding generation of the whole set of paths instead of just one, making the loop over $\mathbf{s}[i]$ parallel, leads to extreme memory consumption as well as unequal distribution of load among nodes. The number of numeric values in the set of paths equals to $N_{\text {Monte Carlo }} \times s[i]$. Simulations, performed in Mazur et al. (2020) showed that normal distribution is attained by estimators at $s=10^{3}$. Given the fact that we need at least $10^{5}$ Monte Carlo trials for a neat histogram of a distribution, one can obtain the amount of memory required to store a matrix of size $N_{\text {Monte Carlo }} \times s[i]$, which makes $763 \mathrm{Mb}$, while some estimators require $80 \mathrm{~Gb}$ per node. That is the reason why in the current version of MCestimLFSM the loop over $\mathrm{s}$ is sequential, and the one over NmonteC is parallel.

During the second part, averages and standard deviations of the samples are computed, and subsequently used to compute the standardized empirical distributions. So that, the three characteristics naturally come together within the same numerical procedure. So far there is no empirical evidence that parallel execution in this section makes MCestimLFSM more efficient.

Such architecture is of great use when the number of nodes available for computations exceeds the number of path length, and the length $s[i]$ differs significantly from $s[j]$ when $i \neq j$.

\section{On some of the other basic functions}

In this part, we will describe aspects of some of the other $\mathrm{R}$ functions implemented in the package.

\section{Higher-order increments}

These increments are the main building block for all statistics we use (see Section 2.3). They are defined as $k$-th iterated increments of step $r$ of a sample path. In particular, $\Delta_{i, 1}^{n, 1} X:=X_{\frac{i}{n}}-X_{\frac{i-1}{n}}$, and $\Delta_{i, 2}^{n, 1} X:=X_{\frac{i}{n}}-2 X_{\frac{i-1}{n}}+X_{\frac{i-2}{n}}$. In rlfsm, we built two functions for computation of objects of this class- increment() and increments(). The former accepts a vector of points at which a user wants to evaluate higher-order increments, and computes them using formula

$$
\Delta_{i, k}^{n, r} X:=\sum_{j=0}^{k}(-1)^{j}\left(\begin{array}{c}
k \\
j
\end{array}\right) X_{(i-r j) / n} .
$$

Before evaluation of (4), the function checks the condition $i<k r$. Evaluation of the increments on a sample path of length $N$ takes $(k+1)(N-k r)$ operations- $k+1$ sums for $N-k r$ points. increments () computes increments iteratively on the whole set of path points. The first iteration gives $N-r$ increments, the second- $N-2 r$ and so on. Thus, the total number of performed 


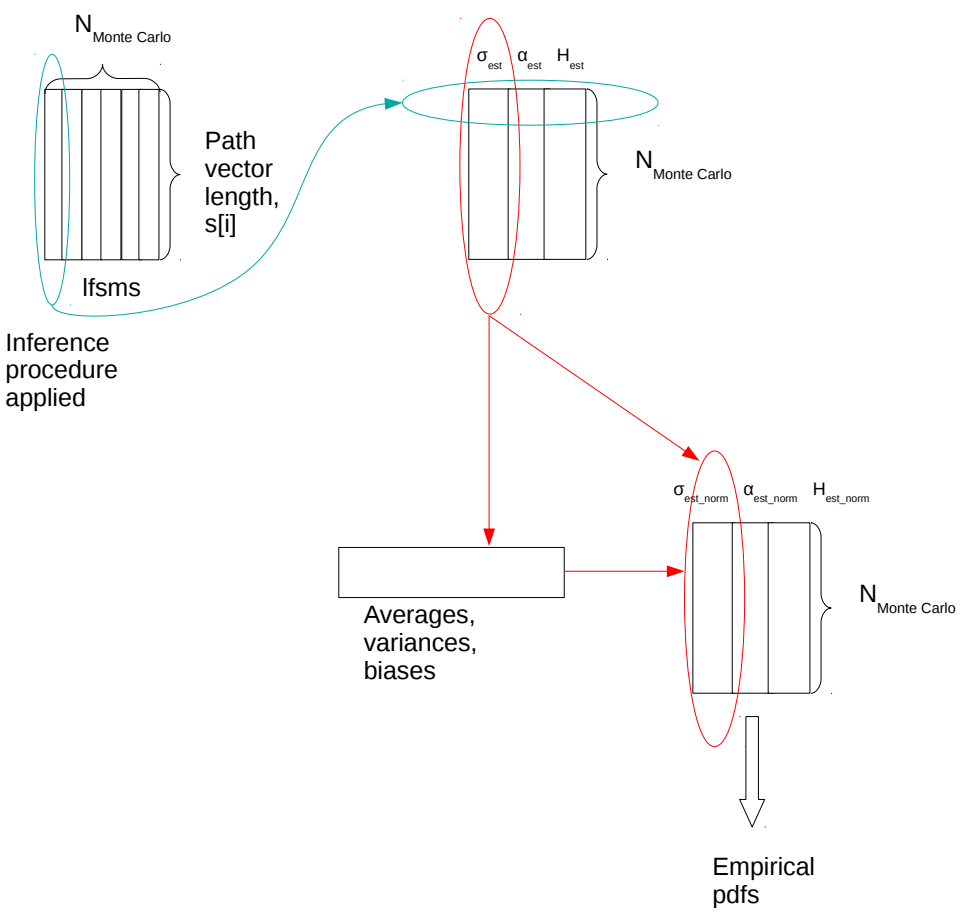

Figure 9: Scheme of extracting estimator statistics by function MCestimLFSM for a chosen path length.

operations is

$$
\sum_{j=1}^{k}(N-j r)=k N-r(k+1) k / 2 .
$$

It is clear that increments () is faster on sample paths with large number of points, but slower when the increment order is high. As we will show later, orders greater than $\sim 10$ are not usable for statistical inference. That is the reason why in all statistics we use either increments () or its hidden "relatives".

\section{A visualization method for sample paths}

We introduce a pair of functions which makes a panel plot of sample paths produced by processes with different parameters. Path_array takes a set of $\alpha-H$ values, generates a path for each combination, and stacks the paths together in a data frame. In the produced data frame all the paths are tagged with $\alpha$ and $H$ values. Plot_list_paths() takes the data frame as an argument and plots the sample paths on different panels based on their $(\alpha, H)$ values. This functionality is powered by facet_wrap() from ggplot2 (Wickham, 2016). For discontinuous paths Plot_list_paths() draws an overlapping semitransparent line joining neighbouring points in order to highlight jumps.

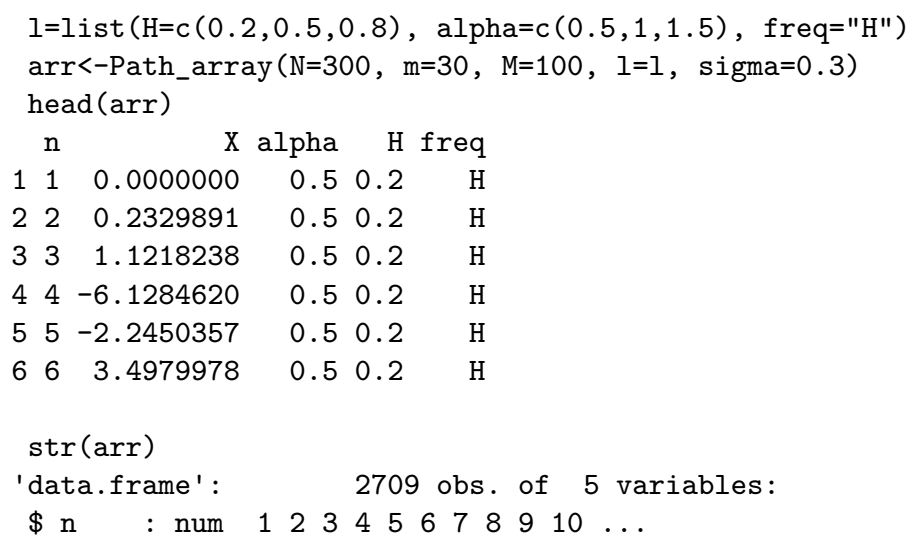



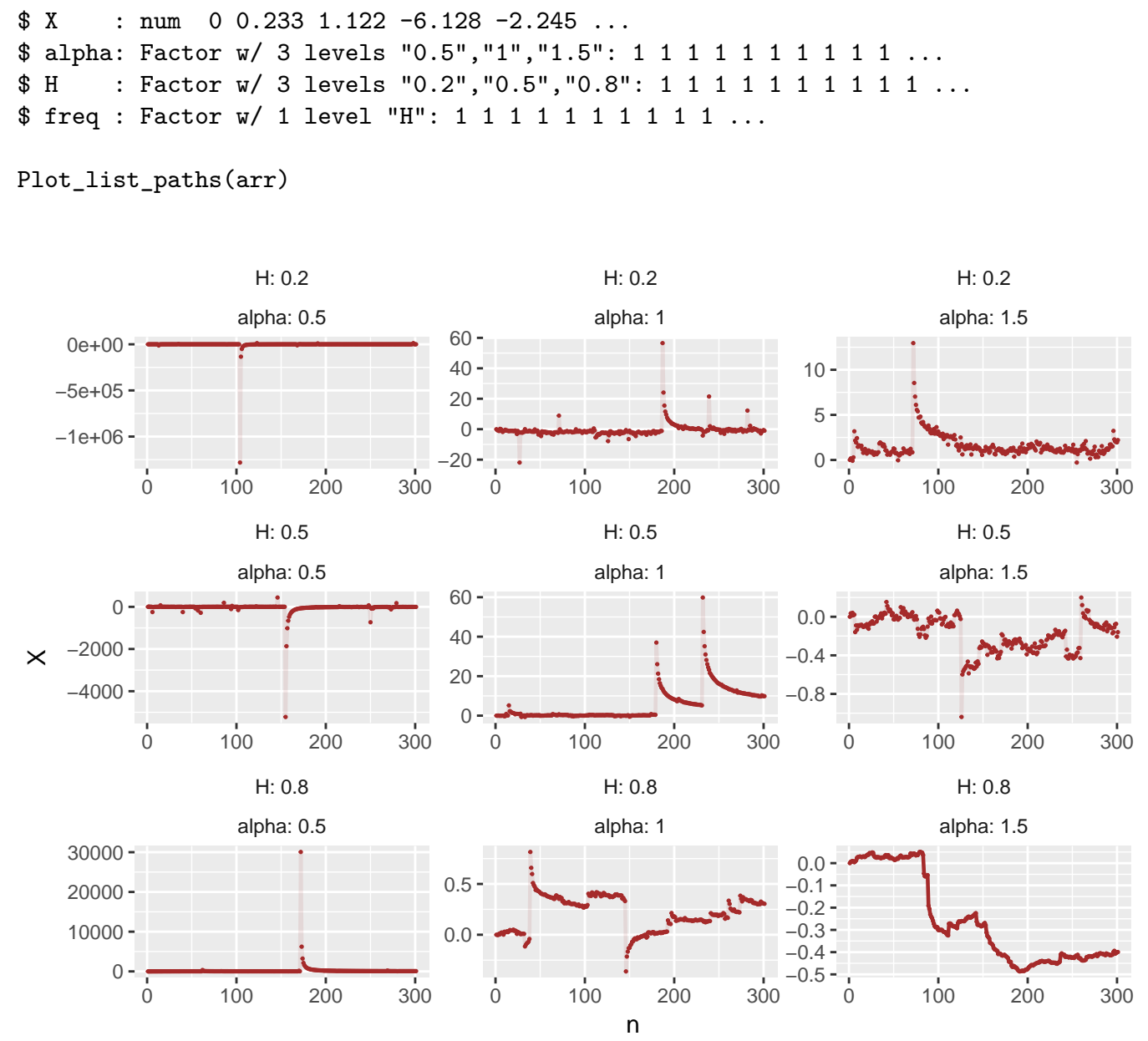

Figure 10: Graph rendered by Plot_list_paths

\section{Parameter estimation of the linear fractional stable motion}

In this section, we describe estimators for the parameters $H, \alpha$, and $\sigma$ that are obtained in the recent paper by Mazur et al. (2020), and their implementation in R.

\section{Parameter estimation in the continuous case}

First, we consider the case $H-1 / \alpha>0$ which leads us to the important property that the lfsm $\left(X_{t}\right)_{t \in R}$ is locally Hölder continuous of any order up to $H-1 / \alpha$. Moreover, this condition implies the following restrictions

$$
\alpha \in(1,2) \text { and } H \in(1 / 2,1)
$$

that allow us to use the law of large numbers in Theorem 1.1 of (Basse-O'Connor et al., 2017) when $p<1$, and the central limit theorem in Theorem 1.2 of (Basse-O'Connor et al., 2017) when $p<1 / 2$, $k \geq 2$ and $H<k-1 / \alpha$.

Now, we consider consistent estimators for the self-similarity parameter $H$ in high- and lowfrequency setting, defined by

$$
\begin{aligned}
\widehat{H}_{\text {high }}(p, k)_{n} & :=\frac{1}{p} \log _{2}\left(\frac{\sum_{i=2 k}^{n}\left|\Delta_{i, k}^{n, 2} X\right|^{p}}{\sum_{i=2 k}^{n}\left|\Delta_{i, k}^{n, 1} X\right|^{p}}\right), \\
\widehat{H}_{\text {low }}(p, k)_{n} & :=\frac{1}{p} \log _{2}\left(\frac{\sum_{i=2 k}^{n}\left|\Delta_{i, k}^{2} X\right|^{p}}{\sum_{i=2 k}^{n}\left|\Delta_{i, k}^{1} X\right|^{p}}\right) .
\end{aligned}
$$


Both estimators for $H$ are based upon a ratio statistic that compares power variations at two different frequencies.

Let us define the following two statistics

$$
V_{h i g h}(f ; k, r)_{n}:=\frac{1}{n} \sum_{i=r k}^{n} f\left(n^{H} \Delta_{i, k}^{n, r} X\right) \quad V_{l o w}(f ; k, r)_{n}:=\frac{1}{n} \sum_{i=r k}^{n} f\left(n^{H} \Delta_{i, k}^{r} X\right),
$$

where $f: \mathbb{R} \rightarrow \mathbb{R}$ is a measurable function. Estimators for the stability index $\alpha$ of the driving stable motion in high and low frequency setting are based on the empirical characteristic functions given by

$$
\varphi_{\text {high }}(t ; H, k)_{n}:=V_{\text {high }}\left(\psi_{t} ; k\right)_{n} \quad \text { and } \quad \varphi_{\text {low }}(t ; k)_{n}:=V_{l o w}\left(\psi_{t} ; k\right)_{n}
$$

with $\psi_{t}(x):=\cos (t x)$, for two different values $t_{1}$ and $t_{2}$ such that $t_{2}>t_{1}>0$. Let us note that the empirical characteristic function $\varphi_{\text {high }}(t ; H, k)_{n}$ depends on the parameter $H$ while $\varphi_{\text {low }}(t ; k)_{n}$ does not. Thus, we should infer the self-similarity parameter $H$ by $\widehat{H}_{h i g h}(p, k)_{n}$ and then we should use the plug-in estimator $\varphi_{h i g h}\left(t ; \widehat{H}_{h i g h}(p, k)_{n}, k\right)_{n}$ to infer the stability index $\alpha$ in high-frequency setting. Estimators for the parameter $\alpha$ are given by

$$
\begin{aligned}
\widehat{\alpha}_{\text {high }} & :=\frac{\log \left|\log \varphi_{\text {high }}\left(t_{2} ; \widehat{H}_{\text {high }}(p, k)_{n}, k\right)_{n}\right|-\log \left|\log \varphi_{\text {high }}\left(t_{1} ; \widehat{H}_{\text {high }}(p, k)_{n}, k\right)_{n}\right|}{\log t_{2}-\log t_{1}}, \\
\widehat{\alpha}_{\text {low }} & :=\frac{\log \left|\log \varphi_{\text {low }}\left(t_{2} ; k\right)_{n}\right|-\log \left|\log \varphi_{\text {low }}\left(t_{1} ; k\right)_{n}\right|}{\log t_{2}-\log t_{1}} .
\end{aligned}
$$

Estimators for the scale parameter $\sigma$ in high- and low-frequency are also based on the empirical characteristic functions which are defined for one value of $t>0$. Further, we define a function $h_{k, r}: R \rightarrow R$ as follows:

$$
h_{k, r}(x)=\sum_{j=0}^{k}(-1)^{j}\left(\begin{array}{l}
k \\
j
\end{array}\right)(x-r j)_{+}^{H-1 / \alpha}, \quad x \in R,
$$

where $k, r \in N$, and let $\left\|h_{k, r}\right\|_{\alpha}^{\alpha}:=\int_{R}\left|h_{k, r}(s)\right|^{\alpha} d s$. Let us note that the function $h_{k, r}$ depends on two parameters $\alpha$ and $H$ which need to be pre-estimated. Estimators for the parameter $\sigma$ are expressed as

$$
\begin{aligned}
\widehat{\sigma}_{\text {high }} & :=\left(-\log \varphi_{\text {high }}\left(t_{1} ; \widehat{H}_{\text {high }}(p, k)_{n}, k\right)\right)^{1 / \widehat{\alpha}_{\text {high }}} / t_{1}\left\|h_{k, 1}\right\|_{\widehat{\alpha}_{\text {high }}}, \\
\widehat{\sigma}_{\text {low }} & :=\left(-\log \varphi_{\text {low }}\left(t_{1} ; k\right)\right)^{1 / \widehat{\alpha}_{\text {low }}} / t_{1}\left\|h_{k, 1}\right\|_{\widehat{\alpha}_{\text {low }}} .
\end{aligned}
$$

\section{Parameter estimation in the general case}

Here, we consider general case when an explicit lower bound for $\alpha$ is unknown. First, we consider estimators which are obtained in low frequency setting. Consistent estimator for parameter $H$ for any $p \in(1,1 / 2)$ is obtained by

$$
\widehat{H}_{\text {low }}(-p, k)_{n}:=\frac{1}{p} \log _{2}\left(\frac{\sum_{i=2 k}^{n}\left|\Delta_{i, k}^{2} X\right|^{-p}}{\sum_{i=2 k}^{n}\left|\Delta_{i, k}^{1} X\right|^{-p}}\right) .
$$

Next, we consider two-step procedure to choose the order of increments $k$, since we should be in the domain of attraction of Theorem 1.2 of (Basse-O'Connor et al., 2017) that requires $k>H+1 / \alpha$. That's why we consider the preliminary estimator of $\alpha$ with $k=1$ that is consistent given by

$$
\widehat{\alpha}_{\text {low }}^{0}\left(t_{1}, t_{2}\right)_{n}=\frac{\log \left|\log \varphi_{\text {low }}\left(t_{2} ; 1\right)_{n}\right|-\log \left|\log \varphi_{\text {low }}\left(t_{1} ; 1\right)_{n}\right|}{\log t_{2}-\log t_{1}} .
$$

Since we do not know if $\widehat{\alpha}_{\text {low }}^{0}\left(t_{1}, t_{2}\right)_{n}$ is in the domain of attraction, we define the estimator of the parameter $k$ as

$$
\hat{k}_{\text {low }}\left(t_{1}, t_{2}\right)_{n}:=2+\left\lfloor\widehat{\alpha}_{\text {low }}^{0}\left(t_{1}, t_{2}\right)_{n}^{-1}\right\rfloor .
$$


In the second step we use estimator $\hat{k}_{\text {low }}:=\hat{k}_{\text {low }}\left(t_{1}, t_{2}\right)_{n}$ for the estimation of parameters $H, \alpha$ and $\sigma$. In particular, we get the following consistent estimators

$$
\begin{aligned}
\widehat{H}_{\text {low }}\left(-p, \hat{k}_{\text {low }}\right)_{n} & =\frac{1}{p} \log _{2}\left(\frac{\sum_{i=2 \hat{k}_{\text {low }}}^{n}\left|\Delta_{i, \hat{k}_{\text {low }}}^{2} X\right|^{-p}}{\sum_{i=2 \hat{k}_{\text {low }}}^{n}\left|\Delta_{i, \hat{k}_{\text {low }}}^{1} X\right|^{-p}}\right) \\
\tilde{\alpha}_{\text {low }}\left(\hat{k}_{\text {low }} ; t_{1}, t_{2}\right)_{n} & =\frac{\log \left|\log \varphi_{\text {low }}\left(t_{2} ; \hat{k}_{\text {low }}\right)_{n}\right|-\log \left|\log \varphi_{\text {low }}\left(t_{1} ; \hat{k}_{\text {low }}\right)_{n}\right|}{\log t_{2}-\log t_{1}}, \\
\tilde{\sigma}_{\text {low }}\left(\hat{k}_{\text {low }} ; t_{1}, t_{2}\right)_{n} & =\left(-\log \varphi_{\text {low }}\left(t_{1} ; \hat{k}_{\text {low }}\right)\right)^{1 / \tilde{\alpha}_{\text {low }}} / t_{1}\left\|h_{\hat{k}_{\text {low }}, 1}\right\|_{\tilde{\alpha}_{\text {low }} .} .
\end{aligned}
$$

Next, we consider two-stage estimation procedure in the general case in high-frequency setting which is the same as in the low-frequency setting. For $p \in(0,1 / 2)$ we compute $\widehat{H}_{h i g h}(-p)_{n}=$ $\widehat{H}_{h i g h}(-p, 1)_{n}$ and, therefore, we can define the preliminary estimator of $\alpha$ by

$$
\widehat{\alpha}_{h i g h}^{0}\left(p, p^{\prime}\right)_{n}=\phi^{-1}\left(\frac{V_{h i g h}\left(f_{-p^{\prime}}, \widehat{H}_{h i g h}(-p)_{n}\right)_{n}^{p}}{V_{h i g h}\left(f_{-p}, \widehat{H}_{h i g h}(-p)_{n}\right)_{n}^{p^{\prime}}}\right)
$$

with

$$
\phi\left(\widehat{\alpha}_{h i g h}^{0}\left(p, p^{\prime}\right)_{n}\right):=\frac{\left(2 / \widehat{\alpha}_{h i g h}^{0}\left(p, p^{\prime}\right)_{n}\right)^{p-p^{\prime}} a_{-p}^{p^{\prime}} \Gamma\left(p^{\prime} / \widehat{\alpha}_{h i g h}^{0}\left(p, p^{\prime}\right)_{n}\right)^{p}}{a_{-p^{\prime}}^{p} \Gamma\left(p / \widehat{\alpha}_{h i g h}^{0}\left(p, p^{\prime}\right)_{n}\right)^{p^{\prime}}}
$$

where $p, p^{\prime} \in(0,1 / 2)$ such that $p \neq p^{\prime}$, and $V_{h i g h}\left(f_{-p}, \widehat{H}_{h i g h}(-p)_{n}\right)_{n}$ is given in formula (6) with $k=1, f_{-p}(x)=|x|^{-p}$ and preliminary estimator $\widehat{H}_{h i g h}(-p)_{n}$ for the parameter $H$. It is remarkable that $\phi(\cdot)$ is always invertible for all $p \neq p^{\prime}$ (see Dang and Istas (2017)). Consequentially, we can define the estimator of $k$ in high-frequency setting by

$$
\hat{k}_{h i g h}:=\hat{k}_{h i g h}\left(p, p^{\prime}\right)_{n}=2+\left\lfloor\widehat{\alpha}_{h i g h}^{0}\left(p, p^{\prime}\right)_{n}^{-1}\right\rfloor .
$$

Thus, consistent estimators of $H, \alpha$ and $\sigma$, in high-frequency setting are given by

$$
\begin{aligned}
\widehat{H}_{h i g h}\left(-p, \hat{k}_{h i g h}\right)_{n} & =\frac{1}{p} \log _{2}\left(\frac{\sum_{i=2 \hat{k}_{h i g h}}^{n}\left|\Delta_{i, \hat{k}_{h i g h}}^{n, 2} X\right|^{-p}}{\sum_{i=2 \hat{k}_{h i g h}}^{n}\left|\Delta_{i, \hat{k}_{h i g h}}^{n, 1} X\right|^{-p}}\right), \\
\tilde{\alpha}_{h i g h}\left(\hat{k}_{h i g h} ; t_{1}, t_{2}\right)_{n} & =\phi^{-1}\left(\frac{V_{h i g h}\left(f_{-p^{\prime}}, \widehat{H}_{h i g h}\left(-p, \hat{k}_{h i g h}\right)_{n} ; \hat{k}_{h i g h}\right)_{n}^{p}}{V_{h i g h}\left(f_{-p}, \widehat{H}_{h i g h}\left(-p, \hat{k}_{h i g h}\right)_{n} ; \hat{k}_{h i g h}\right)_{n}^{p^{\prime}}}\right), \\
\tilde{\sigma}_{h i g h}\left(\hat{k}_{h i g h} ; p, p^{\prime}\right)_{n} & =\left(\frac{\tilde{\alpha}_{h i g h} a_{-p} V_{h i g h}\left(f_{-p}, \widehat{H}_{h i g h}(-p)_{n}\right)_{n}}{2 \Gamma\left(p / \tilde{\alpha}_{h i g h}\right)}\right)^{-\frac{1}{p}} /\left\|h_{\hat{k}_{h i g h}, 1}\right\|_{\tilde{\alpha}_{h i g h}} .
\end{aligned}
$$

\section{Implementation in $\mathbf{R}$}

We introduce function ContinEstim for performing statistical inference according to Section 2.3.1 when $H-1 / \alpha>0$.

ContinEstim(t1, t2, $p, k$, path, freq)

The function is basically comprised by simpler functions alpha_hat, $\mathrm{H}_{-}$hat and sigma_hat responsible for retrieving the corresponding parameters. sigma_hat is called using tryCatch as the former may return an error due to numerical integration in Norm_alpha.

General low-frequency estimation technique, described in Section 2.3.2 is implemented in GenLowEstim.

GenLowEstim(t1, t2, p, path, freq = "L")

This estimator first sets a preliminary $k$ to be equal to 1 , and uses it to compute preliminary parameters $H_{0}$ and $\alpha_{0}$. Using these $H_{0}$ and $\alpha_{0}$, a new $k$ is obtained through 2+floor (alpha_ $\left.0 \hat{(}-1\right)$ ), and then the new $k$ is used for the same estimation procedure as in ContinEstim. This approach induces an effect, which does not exist in the case when ContinEstim is applied. When $\alpha$ is smaller than, or close to $2 / N$, where $N$ is the observed lfsm path length, the computational errors are more frequent. These extra errors occur when the preliminary estimation of $\mathrm{k}$ appears to exceed $N / 2$, making it impossible to compute $\Delta_{i, \hat{k}_{l o w}}^{2} X$ in statistic $\widehat{H}_{\text {low }}\left(-p, \hat{k}_{\text {low }}\right)_{N}$. In case of other sample 
path realizations $k<H+1 / \alpha$, and it is still possible to obtain the estimates which happen to converge to the true value $(\widehat{H}, \widehat{\alpha}, \widehat{\sigma})$, because in this case one would be in the domain of attraction of Theorem 2.2 of (Mazur et al., 2020). Though, the limiting distribution is not stable anymore, and the rate of convergence depends on $\alpha$ and $H$. Real distributions of estimates in this case are left unexplored.

High-frequency estimator from the same section was implemented in GenHighEstim.

GenHighEstim(p, p_prime, path, freq, low_bound $=0.01$, up_bound $=4$ )

\section{Estimate deterioration}

Although the general high- and low-frequency estimators presented in Section 2.3.2 have important advantages, namely closed form expressions for distribution functions and non-suboptimal convergence rates, they also reveal two drawbacks in performance. Due to condition and error handling, the time performances of the general estimators are much worse than those of the continuous ones. On top of that, the plug-in estimators (because of their nature) have much less probability of obtaining an estimate at all. The main idea is as follows: the more statistics are used in a plug-in estimator, the higher the probability to stumble upon a numerical error during the estimation procedure. We illustrate this effect by the following experiment, wherein the general high- and low-frequency estimators are compared to the corresponding continuous ones. For each pair from a set of parameters $(H, \alpha)$, NmonteC sample paths of the both frequencies were generated, and to each of them the relevant procedures ContinEstim, GenLowEstim and GenHighEstim were applied (see "Estimate deterioration experiment" in the supplementary materials). Then, the rates of successful computation results were computed. The result of estimation was considered "successful" if during the procedure all three parameters were obtained, no error occurred, and the estimates are meaningful, namely $(\widehat{H}, \widehat{\alpha}) \in(0,1) \times(0,2)$.

This experiment shows (Figures 11a and 11b) that in both high- and low-frequency cases ContinEstim gives much better precision than the corresponding general estimator. The outcome is rigorous in low-frequency technique since ContinEstim and GenLowEstim have the same set of tuning parameters. On the other hand, the high-frequency estimators have non-coinciding parameter sets, and thus, without fine tuning, the result is merely intuitive. One could observe that in general estimation near the boundaries of the interval $(\widehat{H}, \widehat{\alpha}) \in(0,1) \times(0,2)$ produces more errors, which is partly due to the fact that near the boundaries it is easier to obtain an estimate outside the interval. Such an estimate is removed by Errfilter function in the experiment.

\section{Zones with different convergence regimes in the low-frequency case}

In order to show how the general low-frequency estimation works in practice, we peform a numerical experiment whose code could be found in section "Zones with different convergence" of the accompanying .R file. We set a constant $\sigma$ and choose two sets of parameters- one for $\alpha$ and one for $H$. Then, for each combination of them a number $N_{m c}=500$ of sample paths is created. All path lengths are set to a constant $N=1000$. To each path we apply several statistics. One of them is $k_{-}$new $<-2+f l o o r\left(a l p h a \_0 \hat{(-1)}\right.$ ) where alpha_0 is obtained via alpha_hat with parameters $k=1$, freq='L' plugged-in. This provides us simulated distribution of $\widehat{k}_{\text {low }}$ (Figure 12). Also, we fix a set $\mathrm{k}_{-}$ind $=\operatorname{seq}(1,8, \mathrm{by}=1)$ and, given a path, for each of these k's extract statistics $\varphi_{\text {low }}\left(t, k=k_{\text {ind }}\right)_{n}$ and $\hat{\alpha}_{\text {low }}\left(t_{1}, t_{2} ; k=k_{\text {ind }}\right)_{n}$, see Figures 13 and 14 .

Three regimes of performance of GenLowEstim (read, the general low-frequency estimator $\left.\widehat{\alpha}_{\text {low }}\left(k, t_{1}, t_{2}\right)_{n}\right)$ are observed. To a large extend, only parameter $\alpha$ determines which regime is in presence.

Due to small variance of $\widehat{\alpha}_{\text {low }}^{0}\left(t_{1}, t_{2}\right)_{n}$ (Figure 14), when $\alpha \in(1,2)$ the estimation $\hat{k}_{\text {low }}\left(t_{1}=\right.$ $\left.1, t_{2}=2\right)_{n}$ returns 2 except from the boundaries, where edge effects are observed. This results in the fact that in cases when statistics $\widehat{k}_{\text {low }}(1,2)_{n}$ can be computed without stumbling on numerical errors performances of GenLowEstim and low frequency ContinEstim are the same. At the same time, statistic $\widehat{\alpha}_{\text {low }}\left(k, t_{1}, t_{2}\right)_{n}$ is not far from its limit value for $k<3$, that's why the parameter estimation of the LFSM is technically possible by ContinEstim and GenLowEstim at such length of the sample path.

When $\alpha$ is near 1 there is a transition between the regime with values of $\widehat{k}_{\text {low }}(1,2)_{n}$ concentrated at point $k=2$, and the regime where $\widehat{k}_{\text {low }}(1,2)_{n}$ is highly dispersed. This shift is characterized by only two values of $\widehat{k}_{\text {low }}(1,2)_{n}$ : 2 and 3 . Such behavior of the estimated order of increments is due 

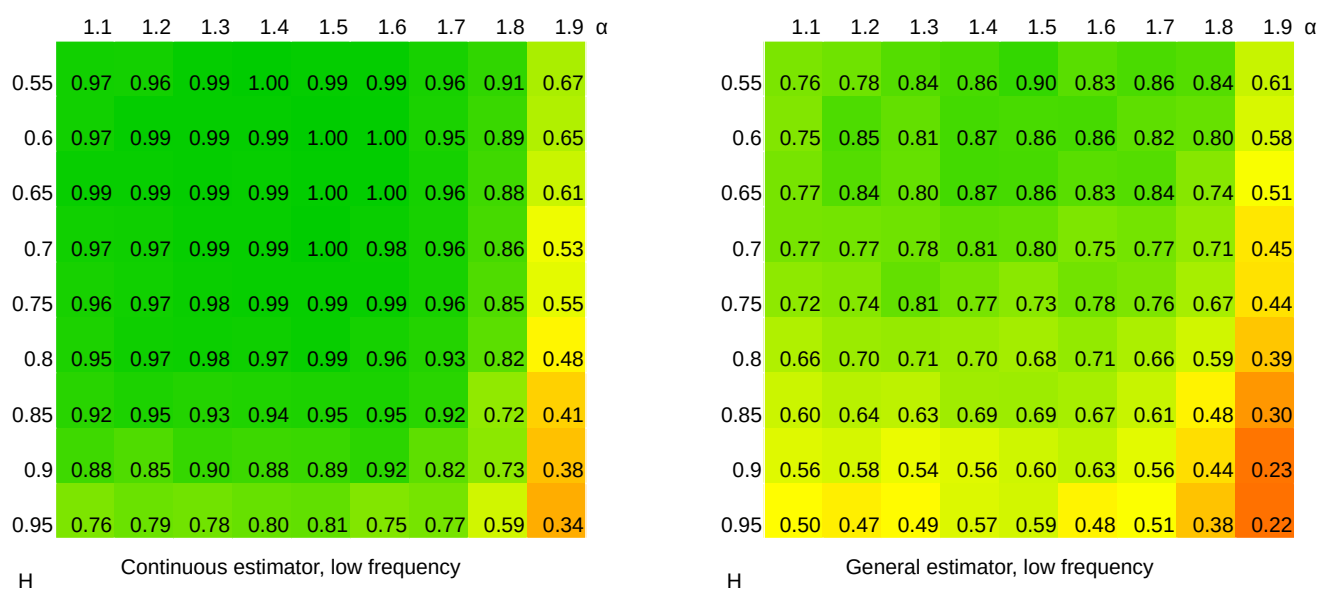

(a) Comparison of success rates for ContinEstim and GenLowEstim. Low frequency case. Path length $\mathrm{N}=200$, number of sample paths NmonteC $=300$.
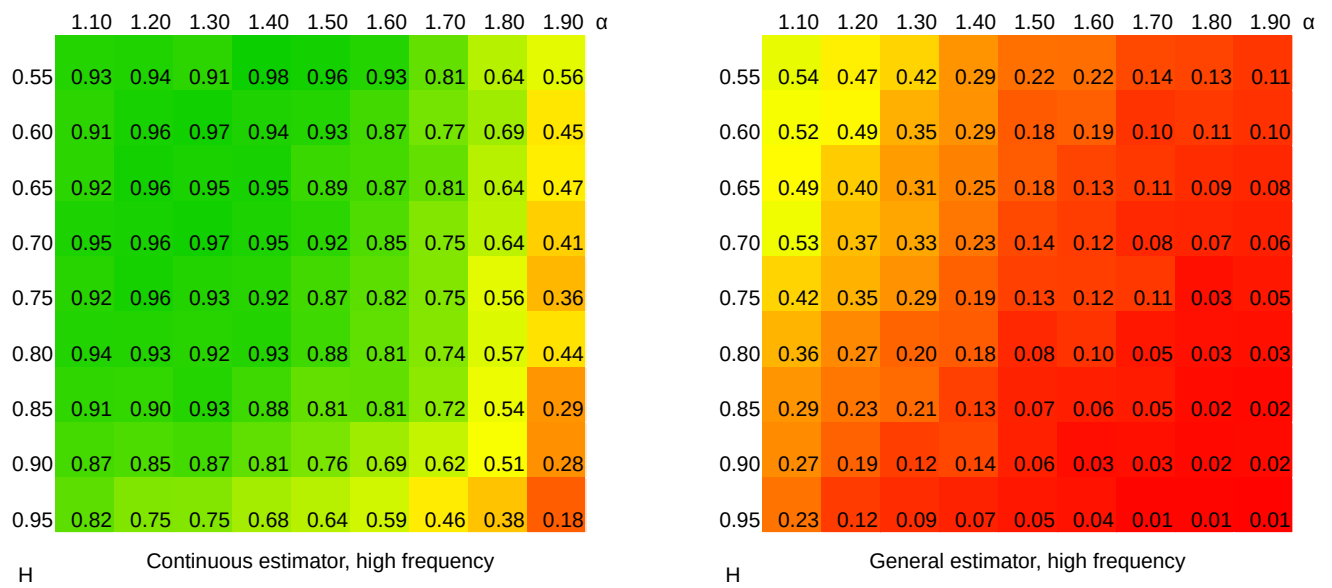

(b) Comparison of success rates for ContinEstim and GenHighEstim. High frequency case. Path length $\mathrm{N}=200$, number of sample paths NmonteC $=300$.

Figure 11: Comparison of success rates of estimators

to the fact that when $\alpha^{-1} \in N$

$$
\mathbb{P}\left(\widehat{k}_{\text {low }}=2+\alpha^{-1}\right) \rightarrow \lambda \quad \text { and } \quad \mathbb{P}\left(\widehat{k}_{\text {low }}=1+\alpha^{-1}\right) \rightarrow 1-\lambda
$$

for some constant $\lambda \in(0,1)$, see Mazur et al. (2020), Section 4.1. Surprisingly, $\lambda$ is close to 0.5 throughout the whole set of $H$ 's (Figure 12). There are no $\widehat{k}_{\text {low }}(1,2)_{n}$ higher than 3 observed because the preliminary estimation of $\alpha$ is still quite precise as one can see from the middle row on Figure 14. After obtaining $\widehat{k}_{\text {low }}(1,2)_{n}$ equal to either 2 or $3, \widehat{\alpha}_{\text {low }}\left(\widehat{k}_{\text {low }}(1,2)_{n}, t_{1}, t_{2}\right)_{n}$ is computed again quite precisely, but worse than in the continuous case.

At $\alpha<1 \widehat{\alpha}_{\text {low }}\left(k, t_{1}, t_{2}\right)_{n}$ has high variance regardless of what $\mathrm{k}$ is chosen, therefore different values are obtained when computing $\widehat{k}_{\text {low }}(1,2)_{n}$. These values plugged-in to $\widehat{\alpha}_{\text {low }}\left(k, t_{1}, t_{2}\right)_{n}$ produce again very dispersed estimates of parameter $\alpha$. This mechanism explains why $\widetilde{\alpha}_{\text {low }}$ has higher variance in discontinuous case $(H-1 / \alpha<0)$ than in continuous (see the numerical study in Section 5 in Mazur et al. (2020)).

The way $\widetilde{\alpha}_{\text {low }}$ behaves could be explained using pic.(13), where $\varphi_{n}$ and $V_{l o w}\left(\psi_{t}, k\right)_{n}$ are plotted. Cases wherein $\widehat{\alpha}_{\text {low }}$ performs poorly coinside with ones wherein $\varphi_{n}$ and $V_{\text {low }}\left(\psi_{t}, k\right)_{n}$ are significantly distant from each other, so convergence $V_{l o w}\left(\psi_{t}, k\right)_{n} \stackrel{a . s .}{\longrightarrow} \varphi_{n}(t ; k)$ isn't observed at the given length of sample paths, which ruins the whole idea of $(\sigma, \alpha)$ estimation. Of course, this effect doesn't affect $H$-estimation because it is based on ratio statistic, which has a different form. 


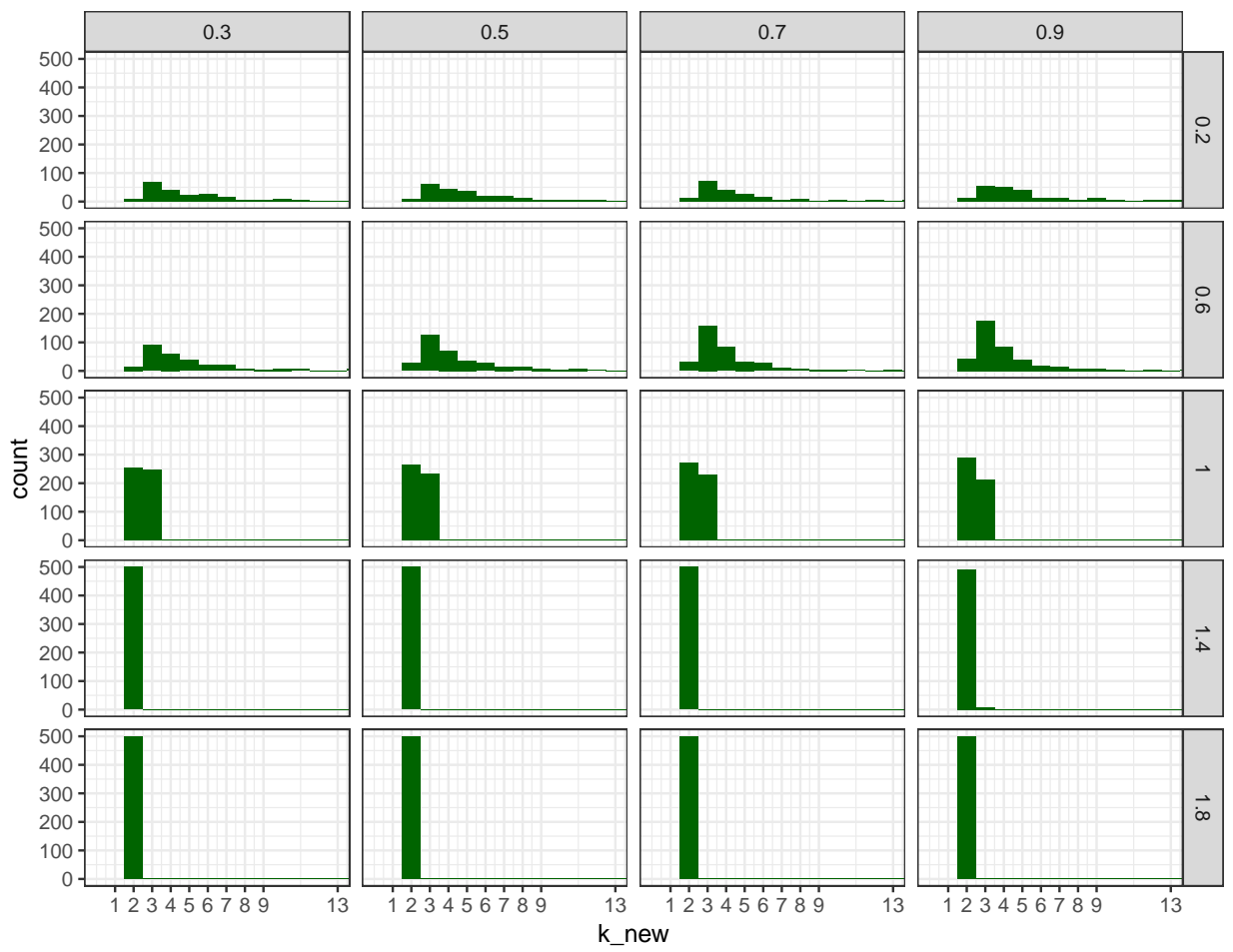

Figure 12: Histograms of preliminary estimations of $\mathrm{k}, \widehat{k}_{\text {low }}(1,2)_{n}$. $\alpha$ 's are on vertical labels, $H$ 'son horizontal.

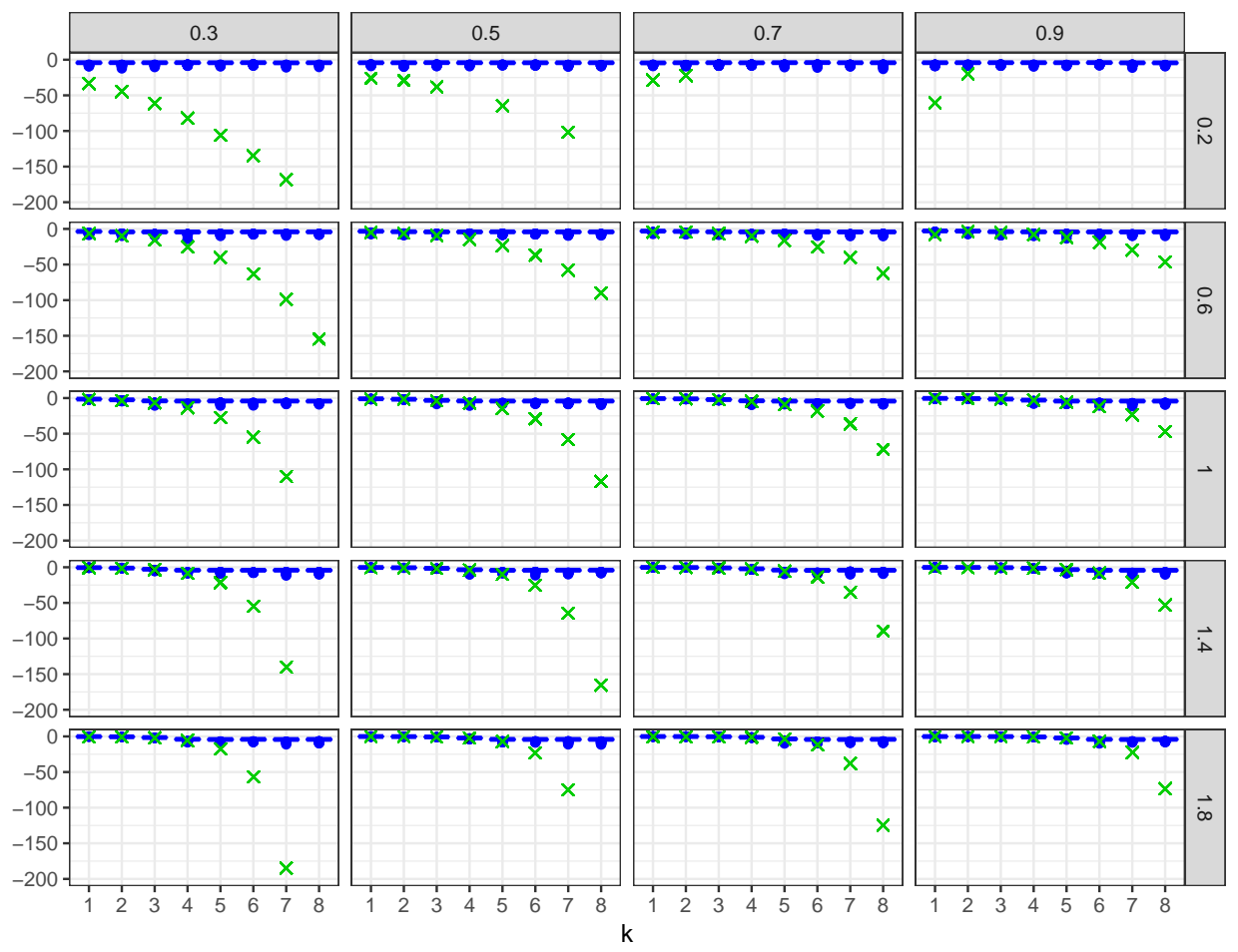

Figure 13: Comparison of the real $\varphi_{n}(t=1 ; k)$ and the one estimated via $V_{l o w}\left(\psi_{t=1}, k\right)_{n}$ on the logarithmic scale. $\alpha$ 's are on vertical labels, $H$ 's- on horizontal. The lower and upper box sides correspond to the 25 th and 75 th percentiles.

\section{S4 classes for Lèvy-driven motions}

Here we describe a simple S4 system (a short introduction to $\mathrm{S} 4$ classes is given in Wickham (2014), Chapter OO field guide) that could be used to simplify manipulations with the two types 


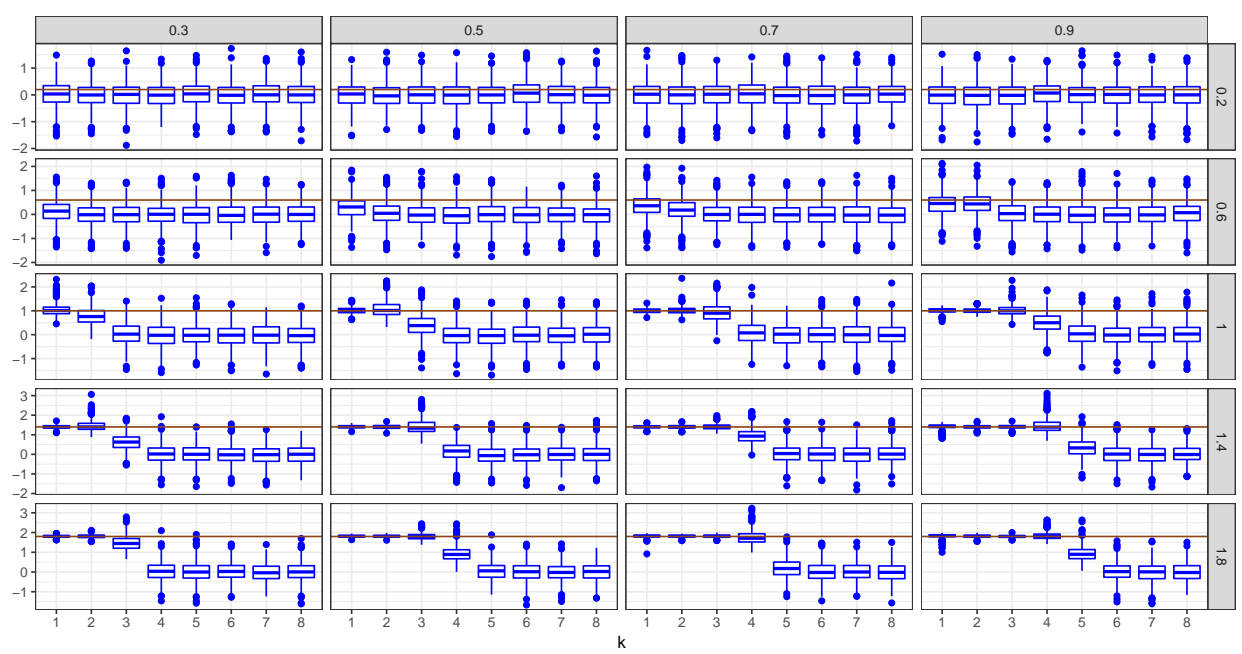

Figure 14: Convergence of $\widehat{\alpha}_{l o w}\left(k, t_{1}, t_{2}\right)_{n}$ to the real $\alpha$ (red line) for different $k$. $\alpha$ 's are on vertical labels, $H$ 's- on horizontal. The lower and upper box sides correspond to the 25 th and 75 th percentiles.

of observations of the linear fractional stable motion. Additionally, we present a possible way to extend the system so that it encompasses more general stochastic processes. The system aims to be helpful in

- passing "attributes" (frequency, $\sigma, \alpha, H$ ) from objects to functions automatically (without additional developer's efforts).

- hiding complicated details of interfaces from users.

- using generics to protract functions on different objects by means of inheritance. For instance, plotting function written for lfsm could be used for other types of stochastic integral.

\section{Classes for simulated lfsm}

Here we describe the least general classes- "SimulatedLfsmLow" and "SimulatedLfsmHigh", objects of which are obtained by simulating low- and high-frequency linear fractional stable motions. Figure

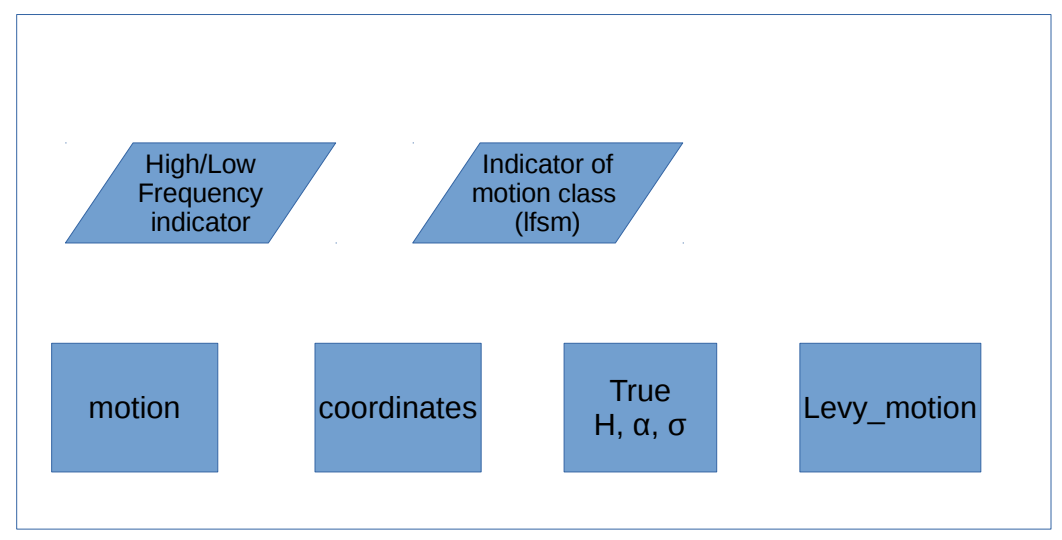

Figure 15: Structure of the classes of simulated lfsm. Frequency indicator and indicator of process type are included in the class name, whilst motion, coordinates, parameters for which the path was simulated and the Lévy motion are written in the slots.

15 shows their internal structure. Roughly speaking, these classes were designed to contain minimum information that could fully describe a simulated LFSM path. Indicators of frequency and a process type are included in the name of a class, which is supposed to make a method dispatch more straightforward, without additional condition blocks. Moreover, all generic functions distinct highand low-frequency schemes of all types with the help of class names. The same holds for motion 
types. Parameters $H, \alpha, \sigma$, as well as Lévy motion, coordinates and the lfsm itself are written in corresponding slots.

\section{Examples}

In the following example we see how an instance of class "SimulatedLfsmLow" is created and then plotting and inference is performed using generic functions plot and ContinInfer. First, we register classes, methods and one generic from "S4 classes examples" in the supplementary materials.
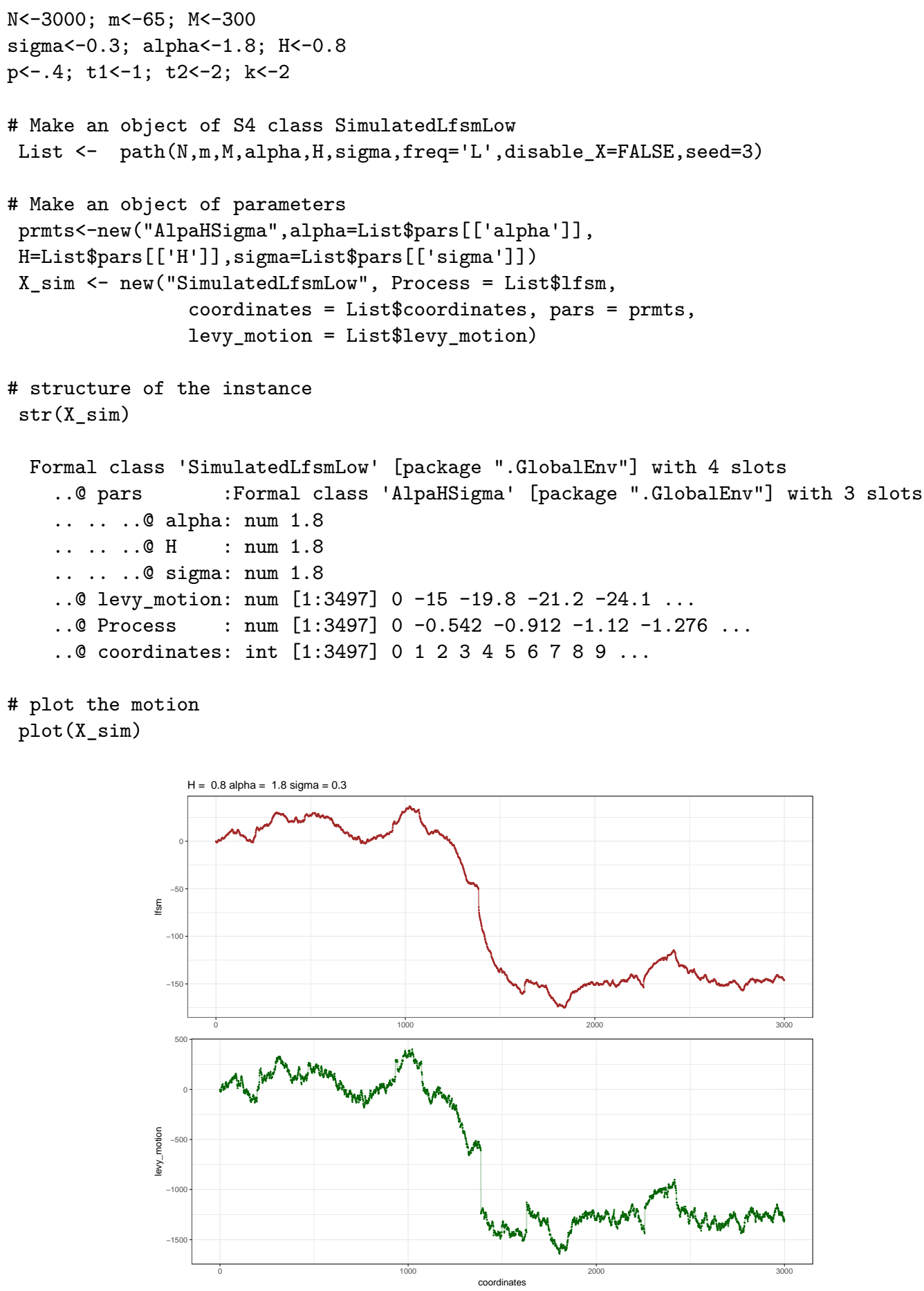

Figure 16: Output of plot method for simulated lfsm

ContinInfer $\left(\mathrm{x}=\mathrm{X}_{-} \mathrm{sim}, \mathrm{t} 1=\mathrm{t} 1, \mathrm{t} 2=\mathrm{t} 2, \mathrm{k}=\mathrm{k}, \mathrm{p}=\mathrm{p}\right)$

\$alpha 
[1] 1.870217

$\$ \mathrm{H}$

[1] 0.8314528

\$sigma

[1] 0.3227219

In this example, the plot function takes almost no effort, compared to the similar one from Section 2.2.2, which is due to the fact, that there has been a method defined for generic plot and object "SimulatedLfsmLow". The last function, ContinInfer, is a generic which has a registered method for class "StochasicProcLow", general stochastic processes in low-frequency setting. Since "SimulatedLfsmLow" inherits from "StochasicProcLow", the generic dispatched this method and performed statistical inference. ContinInfer was designed to perform inference according to Theorem 3.1 from (Mazur et al., 2020) and is based on R function ContinInfer. One can see that plot (and, less obviously, ContinInfer) used "Low" from the name of the class to perform computations.

\section{Acknowledgments}

The authors acknowledge financial support from the project "Ambit fields: probabilistic properties and statistical inference" funded by Villum Fonden. Stepan Mazur acknowledges financial support from the internal research grants at Örebro University, and from the project "Models for macro and financial economics after the financial crisis" (Dnr: P18-0201) funded by Jan Wallander and Tom Hedelius Foundation. The authors would like to thank Prof. Mark Podolskij for significant discussions. Dmitry Otryakhin thanks Dr. Firuza Mamedova for valuable remarks on the draft of this paper.

\section{Bibliography}

A. Basse-O'Connor, R. Lachièze-Rey, and M. Podolskij. Power variation for a class of stationary increments Lévy driven moving averages. Annals of Probability, 45(6B):4477-4528, 2017. URL https://doi.org/10.1214/16-A0P1170. [p396,397]

H. Biermé and H.-P. Scheffler. Fourier series approximation of linear fractional stable motion. Journal of Fourier Analysis and Applications, 14(2):180-202, 2008. URL https://doi.org/10. 1007/s00041-008-9011-7. [p386]

J.-F. Coeurjolly. dvfBm: Discrete variations of a fractional Brownian motion, 2009. URL https: //CRAN.R-project.org/package=dvfBm. R package version 1.0. [p386]

T. Dang and J. Istas. Estimation of the Hurst and the stability indices of a $H$-self-similar stable process. Electronic Journal of Statistics, 11(2):4103-4150, 2017. URL https://doi .org/10.1214/ 17-EJS1357. [p398]

J. Huang. somebm: some Brownian motions simulation functions, 2013. URL https://CRAN.Rproject.org/package=somebm. R package version 0.1. [p386]

S. Mazur, D. Otryakhin, and M. Podolskij. Estimation of the linear fractional stable motion. Bernoulli, 26(1):226-252, 2020. URL https://doi.org/10.3150/19-BEJ1124. [p386, 387, 391, 394, 396, 399, 400, 404]

G. Samorodnitsky and M. S. Taqqu. Stable non-Gaussian random processes: stochastic models with infinite variance, volume 1. CRC Press, 1994. [p386]

S. Stoev and M. Taqqu. Simulation methods for linear fractional stable motion and FARIMA using the fast Fourier transform. Fractals, 95(1):95-121, 2004. URL https://doi.org/10.1142/ S0218348X04002379. [p386, 387, 388]

B. Swihart, J. Lindsey, and P. Lambert. stable: Probability Functions and Generalized Regression Models for Stable Distributions, 2017. URL https://CRAN.R-project.org/package=stable. R package version 1.1.2. [p386]

N. W. Watkins, D. Credgington, R. Sanchez, and S. C. Chapman. A kinetic equation for linear fractional stable motion with applications to space plasma physics. ArXiv e-prints, 2008. URL https://arxiv.org/pdf/0803.2833.pdf. [p386] 
H. Wickham. Advanced R. CRC Press, 2014. URL https://adv-r.hadley.nz/index.html. [p401]

H. Wickham. ggplot2: Elegant Graphics for Data Analysis. Springer-Verlag New York, 2016. ISBN 978-3-319-24277-4. URL https://ggplot2.tidyverse.org. [p395]

W. B. Wu, G. Michailidis, and D. Zhang. Simulating sample paths of linear fractional stable motion. IEEE Transactions on Information Theory, 50(6):1086-1096, 2004. URL https://doi.org/10. 1109/TIT. 2004.828059. [p386]

D. Wuertz, M. Maechler, and Rmetrics core team members. stabledist: Stable Distribution Functions, 2016. URL https://CRAN.R-project.org/package=stabledist. R package version 0.7-1. [p386]

\author{
Stepan Mazur \\ School of Business, Örebro University \\ Fakultetsgatan 1, SE-701 82 Örebro \\ Sweden \\ ORCiD: 0000-0002-1395-9427 \\ stepan.mazur@oru.se \\ Dmitry Otryakhin ${ }^{1}$ \\ Department of Mathematics, Aarhus University \\ Ny Munkegade 118, DK-8000 Aarhus C \\ Denmark \\ ORCiD: 0000-0002-4700-7221 \\ d.otryakhin.acad@protonmail.ch
}

\footnotetext{
${ }^{1}$ some parts of the work were done at the Department of Mathematics of Stockholm University.
} 